BRAVILLIAN JOURNAL

www.bjournal.com.br
ISSN 0100-879X

Volume 44 (8) 729-813 August 2011

BIOMEDICAL SCIENCES

AND

CLINICAL INVESTIGATION

Braz J Med Biol Res, August 2011, Volume 44(8) 810-813

doi: 10.1590/S0100-879X2011007500089

Frequency of primary glomerular disease in northeastern China

Yi-Qi Wu, Zheng Wang, Hua-Feng Xu, Xiao-Ming Jin and Hai-Zhou Zhou

The Brazilian Journal of Medical and Biological Research is partially financed by

贵CNPq
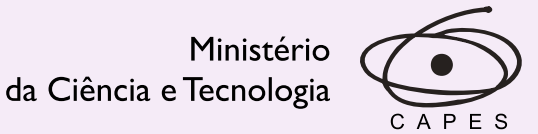

Ministério da Educação
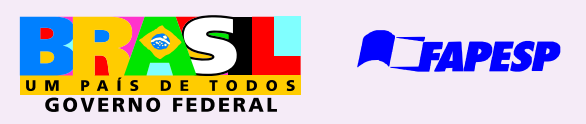
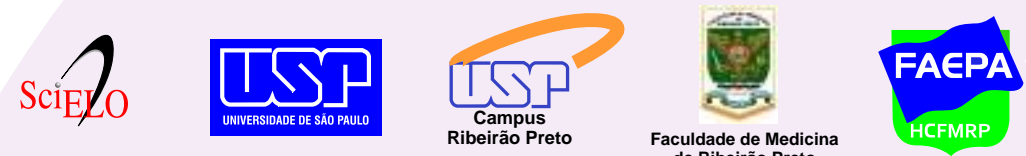

de Ribeirão Preticin
Institutional S ponsors

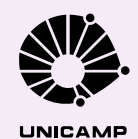

UNICAMP
SHIMADZU

Explore High - Performance MS Orbitrap Technology In Proteomics \& Metabolomics

analitica Thermo 


\title{
Frequency of primary glomerular disease in northeastern China
}

\author{
Yi-Qi Wu ${ }^{1 *}$, Zheng Wang ${ }^{3 *}$, Hua-Feng Xü2,4, Xiao-Ming Jin $^{1}$ and Hai-Zhou Zhou ${ }^{5}$ \\ ${ }^{1}$ Department of Pathology, ${ }^{2}$ Genome Center, Harbin Medical University, Harbin, Heilongjiang, China \\ ${ }^{3}$ Department of Cardiology, Hainan Province Nongken Sanya Hospital, Sanya, Hainan, China \\ ${ }^{4}$ Life Science, Heilongjiang University, Harbin, Heilongjiang, China \\ ${ }^{5}$ Department of Laboratory Diagnosis, the First Affiliated Hospital of Harbin Medical University, \\ Harbin, Heilongjiang, China
}

\begin{abstract}
Most frequently reported Chinese renal biopsy data have originated from southeastern China. The present study analyzed the renal biopsy data from northeastern China. The records of 1550 consecutive native patients who were diagnosed with primary glomerular diseases (PGD) after renal biopsy at our hospital during 2005-2009 were used. These patients were divided into four age groups for stratified analysis: $<15,15-44,45-59$, and $\geq 60$ years old. Among PGD, minimal change disease (MCD) was the most common histologically diagnosed disease (30.7\%), followed by IgA nephropathy (IgAN), mesangial proliferative glomerulonephritis (MsPGN), membranous nephropathy (MN), membranoproliferative glomerulonephritis (MPGN), focal segmental glomerulosclerosis (FSGS), and endocapillary proliferative glomerulonephritis (EnPGN). MCD was the disease most frequently observed $(43.7 \%)$ in the $<15$-year-old group. MsPGN was the most common disease in the elderly group (38.1\%). MsPGN was more prevalent in females (27.8\%), whereas MCD was more prevalent in males (35.3\%). Primary glomerular diseases constituted the most commonly encountered group of diseases with a high prevalence of MCD, which predominantly affected males and young adults. The prevalence of MCD was high in northeastern China. Further study is necessary to expand the epidemiologic data available for renal disease in China.
\end{abstract}

Key words: Primary glomerular disease; Renal biopsies; IgA nephropathy; China

\section{Introduction}

Chronic kidney disease (CKD) is a worldwide public health problem with increasing incidence and prevalence, poor outcome and high associated costs (1). Glomerulonephritis and hypertension are the most common causes of CKD in developing countries. Primary glomerular disease (PGD) is the leading cause of CKD in China $(2,3)$. Common causes of PGD include minimal change nephrotic syndrome (minimal change disease, MCD) and its variants, membranous glomerulonephritis, or membranoproliferative glomerulonephritis (MPGN) (4). The patterns of PGD differ by country and are also changing over time within countries. These patterns are probably changing because of better infection control, changes in environmental pollution, increasing awareness of the disease, and increases in life expectancy (5). In studies from East Asia, IgA nephropathy (IgAN) and non-IgA mesangial proliferative glomerulonephritis (MsPGN) are the most common types of PGD. In most European studies, IgAN and membranous nephropathy (MN) are the common types of PGD. In the USA, the incidence of focal segmental glomerulosclerosis (FSGS), which appears to be the most frequent PGD type, is increasing $(6,7)$. A renal biopsy is required for a definitive diagnosis and has been performed routinely in China for the last 20 years. Most reported renal biopsy data originate from southeastern China, which includes Shanghai, Nanjing and Hong Kong (4,8-10). Epidemiologic PGD data for the northeastern region are rarely reported. This article presents the epidemiological data from renal biopsies performed in Heilongjiang Province (east longitude 121 $13^{\prime}-135^{\circ} 05^{\prime}$; north latitude $\left.43^{\circ} 22^{\prime}-53^{\circ} 24^{\prime}\right)$. Because many patients reside in different regions of the country, these data might supplement the previously published data related to the

Correspondence: Hai-Zhou Zhou, Department of Laboratory Diagnosis, the First Affiliated Hospital of Harbin Medical University, 23 Youzheng Street, Nangang District, Harbin, Heilongjiang 150001, China. E-mail: haizhouzhou@yahoo.com.cn or Xiao-Ming Jin, Department of Pathology, Harbin Medical University, 194 Xuefu Road, Harbin, Heilongjiang 150081, China. E-mail: Jinxm2010@126.com

*These authors contributed equally to this study.

Received January 4, 2011. Accepted July 4, 2011. Available online July 15, 2011. Published August 19, 2011. 
epidemiology of renal disease in China.

\section{Material and Methods}

Patients
The medical records of 1550 patients with PGD diagnosed by renal biopsy were collected from the First Clinical Medical College, Harbin Medical University, Heilongjiang Province, during the period from January 2005 to December 2009. Subjects resided in all 13 regions of Heilongjiang Province $\left(454,000 \mathrm{~km}^{2}\right)$. Of the 1550 patients, $728(47.0 \%)$ were males, and $822(53.0 \%)$ were females. Their average age was $35.1 \pm 12.5$ ( 6 to 75 ) years. The indications for renal biopsy were as follows: 1) nephrotic syndrome (NS; proteinuria $\geq 3.5 \mathrm{~g} /$ day with or without hypoalbuminemia); 2 ) chronic nephritic syndrome (CGN; proteinuria 1-3.5 g/day and a >3-month history of hematuria); 3) acute nephritic syndrome (AGN; hematuria, red blood cell casts and a <3-month history of proteinuria $<3.5 \mathrm{~g} /$ day); 4) rapidly progressive glomerulonephritis syndrome (RPGN; AGN with acute deterioration of renal function, such as a 2-fold increase in the serum creatinine concentration or a $50 \%$ decrease in creatinine clearance), and 5) asymptomatic urinary abnormality (AUA; proteinuria $<1.0 \mathrm{~g} /$ day and hematuria found in routine check-ups, without edema, hypertension or abnormal renal function). The indications for a renal biopsy did not change during the entire observation period. All biopsy specimens were prepared and examined by the same group of clinicians and technicians. As previously described, the analysis included light microscopy, immunohistology, and electron microscopy (3). Renal biopsy specimens were fixed with $10 \%$ neutral buffer formalin and embedded in paraffin using standard procedures. In addition, 2-mm thick sections were stained with hematoxylin and eosin (HE), periodic acid-Schiff reagent (PAS), silver methenamine, and Masson's trichrome. Immunofluorescence techniques were performed using fluorescence-labeled rabbit anti-human $\lg \mathrm{G}, \lg \mathrm{M}, \lg \mathrm{A}, \mathrm{C} 3, \mathrm{C} 4$, and $\mathrm{C} 1 \mathrm{q}$ antibodies. All specimens were examined by two experienced pathologists. A final diagnosis was made for each patient on the basis of the clinical and histological investigations.

The pathological classification of PGD was as follows: IgAN, MsPGN, MN, FSGS, endocapillary proliferative glomerulonephritis (EnPGN), MCD, and MPGN.

\section{Statistical analysis \\ Data were stored in a database file (Excel 2007). All statistical} liferative glomerulonephritis. analyses were performed using the SPSS software (SPSS 11.0, USA). Categorical variables were analyzed using chisquare and Fisher exact tests. Continuous variables were analyzed using the Student $t$-test. Statistical significance was defined as $\mathrm{P}<0.05$.

\section{Results}

\section{Spectrum of PGD}

In the 1550 PGD patients, the leading cause of PGD was MCD ( $\mathrm{N}=476,30.7 \%)$, followed by $\lg A \mathrm{~N}(\mathrm{~N}=380$, $24.5 \%)$, MsPGN (N=366, 23.8\%), MN (N=170, 11.0\%), MPGN $(\mathrm{N}=90,5.8 \%)$, FSGS $(\mathrm{N}=62,4.0 \%)$, and EnPGN $(\mathrm{N}=6,0.4 \%)$.

\section{The clinicopathologic correlations of PGD}

The most common cause of NS was MCD (49.1\%), followed by MsPGN (23.5\%), MN (10.6\%), and IgAN (9.1\%). The most common cause of RPGN syndrome was MPGN $(87.5 \%)$, followed by FSGS (12.5\%). IgAN was the leading cause of AUA, AGN and CGN, accounting for 78.9, 59.1, and $26.9 \%$ of IgAN cases, respectively.

\section{The prevalence of PGD in different age groups}

The distribution of PGD according to patient age is presented in Table 1. There were 32, 1158, 318, and 42 patients with PGD in the $<15,15-44,45-59$, and $\geq 60$-yearold groups, respectively. In the $<15$-year-old group, a high incidence of MCD was identified (43.7\%; P<0.01), followed by $\lg A N(18.7 \%)$, MsPGN (12.5\%), FSGS (12.5\%), MN (6.3\%), and EnPGN (6.3\%). In the 15-44-year-old group, there was no significant difference between the proportions of MCD (29.9\%), IgAN (28.7\%), and MsPGN (24.0\%). In this age group, there were smaller proportions of $\mathrm{MN}(8.1 \%)$, MPGN $(5.5 \%)$, FSGS (3.6\%), and EnPGN $(0.2 \%)$. In the 45-59-year-old group, MCD accounted for 32.7\%, MsPGN for $22.0 \%$, MN for $20.1 \%$, IgAN for $11.9 \%$, MPGN for $7.5 \%$, FSGS for $5.0 \%$, and EnPGN for $0.6 \%$ of PGD cases. Finally, in the $\geq 60$-year-old group, MsPGN was the most common form of PGD (38.1\%), followed by MN (23.8\%),

Table 1. Frequency of primary glomerular diseases according to age.

\begin{tabular}{lccccccc}
\hline Age (years) & IgAN (\%) & MsPGN (\%) & MN (\%) & FSGS (\%) & EnPGN (\%) & MCD (\%) & MPGN (\%) \\
\hline$<15(\mathrm{~N}=32)$ & 18.7 & 12.5 & 6.3 & 12.5 & 6.3 & 43.7 & 0 \\
$15-44(\mathrm{~N}=1158)$ & 28.7 & 24.0 & 8.1 & 3.6 & 0.2 & 29.9 & 5.5 \\
$45-59(\mathrm{~N}=318)$ & 11.9 & 22.0 & 20.1 & 5.0 & 0.6 & 32.7 & 7.5 \\
$\geq 60(\mathrm{~N}=42)$ & 9.5 & 38.1 & 23.8 & 0 & 0 & 23.8 & 4.8 \\
Total $(\mathrm{N}=1550)$ & 24.5 & 23.8 & 11.0 & 4.0 & 0.4 & 30.7 & 5.8 \\
\hline
\end{tabular}

Data are reported as percent. IgAN = IgA nephropathy; MsPGN = mesangial proliferative glomerulonephritis; $\mathrm{MN}=$ membranous nephropathy; FSGS = focal segmental glomerulosclerosis; EnPGN = endocapillary proliferative glomerulonephritis; MCD = minimal change disease; MPGN = membranopro- 
$\operatorname{MCD}(23.8 \%)$, IgAN (9.5\%), and MPGN (4.8\%).

\section{The prevalence of PGD in males and females}

As shown in Table 2, the proportions of MsPGN and MCD differed between males and females. MsPGN was more prevalent among females $(27.8 \%)$ than among males (18.9\%; $P<0.01)$ and MCD was more prevalent among males $(35.3 \%)$ than among females $(26.6 \%$; $P<0.01)$. Among females, there was no significant difference between the proportions of MCD, IgAN and MsPGN $(26.6,25.4$, and $27.8 \%$, respectively).

\section{Discussion}

In the present study, we analyzed the clinical and pathological data of 1550 patients who were diagnosed with PGD between 2005 and 2009 in northeastern China. We found that MCD was the most common form of PGD (30.7\%), followed by IgAN, MsPGN, MN, FSGS, and MPGN.

MCD is diagnosed when a patient has nephrotic syndrome. The renal biopsy reveals little or no change in the structure of the glomeruli or the surrounding tissues as assessed by light microscopy. MCD has a variable geographic distribution and is more common in Asia than in North America or Europe (11). In Korea (12) and Thailand (13), MCD accounts for 26.6 and $45.8 \%$, respectively, of PGD. MCD may occur at any age, but is most common in childhood (14). The predominance of young adults has also been observed in Asian studies of patients with a mean age of 27-31 years $(15,16)$. In addition, a slight male predominance is noted more frequently among Asian patients (male:female $=1.2-2.0: 1)$ than among Western patients (male:female $=0.5-1.3: 1)(17)$. In our study, MCD was the most common PGD in the < 15-year-old group (43.7\%). The mean age of adult-onset MCD was relatively younger (30.9 years) compared to the other PGD forms (30.9 years), and $80 \%$ of patients were less than 40 years old. The male-tofemale ratio of MCD patients was 1.33 , the same value reported in previous studies.

IgA nephropathy is one of the most common forms of glomerulonephritis, accounting for approximately $45 \%$ of primary glomerulonephritis cases in China (3). Few epidemiologic studies have examined the incidence of primary IgA nephropathy in various populations in the world. There is a marked regional difference in the frequency of IgA nephropathy among PGD patients. IgAnephropathy occurs with the highest frequency in Asian countries (Japan and China) $(3,8,18)$ and is less common in North America and Europe (USA and Italy) $(19,20)$. Genetic factors are thought to underlie the development of IgAN in Asia because the incidence of IgAN is much higher in Asia than in Western
Table 2. Gender distribution of primary glomerular diseases.

\begin{tabular}{lrcc}
\hline Pathologic diagnosis & $\mathrm{N}$ & Male & Female \\
\hline IgAN & 380 & $170(23.6)$ & $210(25.4)$ \\
MsPGN & 366 & $138(18.9)$ & $228(27.8)$ \\
MN & 170 & $84(11.5)$ & $86(10.5)$ \\
FSGS & 62 & $34(4.7)$ & $28(3.4)$ \\
EnPGN & 6 & $2(0.3)$ & $4(0.5)$ \\
MCD & 476 & $258(35.3)$ & $218(26.6)$ \\
MPGN & 90 & $42(5.8)$ & $48(5.9)$ \\
\hline
\end{tabular}

Data are reported as number, with percent in parentheses. IgAN = IgA nephropathy; MsPGN = mesangial proliferative glomerulonephritis; $\mathrm{MN}=$ membranous nephropathy; FSGS = focal segmental glomerulosclerosis; EnPGN = endocapillary proliferative glomerulonephritis; $\mathrm{MCD}=$ minimal change disease; $\mathrm{MPGN}=$ membranoproliferative glomerulonephritis.

countries. In our study, Heilongjiang Province showed a low IgAN prevalence $(24.5 \%)$ compared to other regions of China $(3,8,18)$. Heilongjiang is located in Northeastern China, which is an area with unique geographical features and climate conditions. Winter lasts more than 6 months in this region. Economic growth and standards of living are lower than in the Southeastern region of China, which includes cities such as Shanghai, Nanjing, and Hong Kong. With the improvement in living conditions, people lifestyle and dietary changes may have an impact on the incidence of IgAN.

Our results show that NS was the most common clinical presentation of PGD ( $60.6 \%$ of patients). This finding is consistent with previous studies, in which NS has been demonstrated to be the most common renal syndrome $(6,13)$. However, the clinicopathologic presentations of PGD vary in different regions. In Heilongjiang Province, MCD is the most common cause of NS and IgAN is the leading cause of AUA, AGN, and CGN. Clinical practice shows that the same pathological type can have different clinical manifestations, and vice versa. Although renal biopsy can provide information about the type and severity of renal diseases, the diagnosis of PGD should be closely integrated with clinical and the pathological diagnosis in order to rule out a systemic or genetic disease and to obtain the correct diagnosis.

This report, which is based on data from a 5-year period, provides information on renal diseases diagnosed by renal biopsy. This is the first systematic report that focused on data from northeastern China, and the data presented complement reported data on the epidemiology of renal disease in China. 


\section{References}

1. Levey AS, Coresh J, Balk E, Kausz AT, Levin A, Steffes MW, et al. National Kidney Foundation practice guidelines for chronic kidney disease: evaluation, classification, and stratification. Ann Intern Med 2003; 139: 137-147.

2. Zuo L, Wang M. Current status of hemodialysis treatment in Beijing, China. Ethn Dis 2006; 16: S2-S4.

3. Zhou FD, Zhao MH, Zou WZ, Liu G, Wang H. The changing spectrum of primary glomerular diseases within 15 years: a survey of 3331 patients in a single Chinese centre. Nephrol Dial Transplant 2009; 24: 870-876.

4. Yuen LK, Lai WM, Lau SC, Tong PC, Tse KC, Chiu MC. Ten-year review of disease pattern from percutaneous renal biopsy: an experience from a paediatric tertiary renal centre in Hong Kong. Hong Kong Med J 2008; 14: 348-355.

5. Mitwalli AH, Al Wakeel J, Abu-Aisha H, Alam A, Al Sohaibani $\mathrm{M}$, Tarif $\mathrm{N}$, et al. Prevalence of glomerular diseases: King Khalid University Hospital, Saudi Arabia. Saudi J Kidney Dis Transpl 2000; 11: 442-448

6. Ossareh S, Asgari M, Abdi E, Nejad-Gashti H, Ataipour Y, Aris $S$, et al. Renal biopsy findings in Iran: case series report from a referral kidney center. Int Urol Nephrol 2010; 42: 1031-1040.

7. Woo KT, Chan CM, Chin YM, Choong HL, Tan HK, Foo M, et al. Global evolutionary trend of the prevalence of primary glomerulonephritis over the past three decades. Nephron Clin Pract 2010; 116: c337-c346.

8. Li LS, Liu ZH. Epidemiologic data of renal diseases from a single unit in China: analysis based on 13,519 renal biopsies. Kidney Int 2004; 66: 920-923.

9. Cai GY, Chen XM. Immunoglobulin A nephropathy in China: progress and challenges. Am J Nephrol 2009; 30: 268273.

10. Yao Q, Zhang W, Qian J. Dialysis status in China: a report from the Shanghai Dialysis Registry (2000-2005). Ethn Dis 2009; 19: S1-S6.
11. Sharples PM, Poulton J, White RH. Steroid responsive nephrotic syndrome is more common in Asians. Arch Dis Child 1985; 60: 1014-1017.

12. Choi IJ, Jeong HJ, Han DS, Lee JS, Choi KH, Kang SW, et al. An analysis of 4,514 cases of renal biopsy in Korea. Yonsei Med J 2001; 42: 247-254.

13. Parichatikanond P, Chawanasuntorapoj R, Shayakul C, Choensuchon B, Vasuvattakul S, Vareesangthip K, et al. An analysis of 3,555 cases of renal biopsy in Thailand. $J$ Med Assoc Thai 2006; 89 (Suppl 2): S106-S111.

14. Fogo $A B$. Minimal change disease and focal segmental glomerulosclerosis. Nephrol Dial Transplant 2001; 16 (Suppl 6): 74-76

15. Wang F, Looi LM, Chua CT. Minimal change glomerular disease in Malaysian adults and use of alternate day steroid therapy. Q J Med 1982; 51: 312-328.

16. Nair RB, Date A, Kirubakaran MG, Shastry JC. Minimalchange nephrotic syndrome in adults treated with alternateday steroids. Nephron 1987; 47: 209-210.

17. Huang JJ, Hsu SC, Chen FF, Sung JM, Tseng CC, Wang $\mathrm{MC}$. Adult-onset minimal change disease among Taiwanese: clinical features, therapeutic response, and prognosis. Am J Nephrol 2001; 21: 28-34.

18. Fu KY, Chen R, Zhan F, Cai JH, Xie YY, Wu Y, et al. [Clinical and pathological analysis of 217 patients with IgA nephropathy from Hainan Province]. Nan Fang Yi Ke Da Xue Xue Bao 2009; 29: 1445-1447.

19. Dragovic D, Rosenstock JL, Wahl SJ, Panagopoulos G, DeVita MV, Michelis MF. Increasing incidence of focal segmental glomerulosclerosis and an examination of demographic patterns. Clin Nephrol 2005; 63: 1-7.

20. Schena FP. Survey of the Italian Registry of Renal Biopsies. Frequency of the renal diseases for 7 consecutive years. The Italian Group of Renal Immunopathology. Nephrol Dial Transplant 1997; 12: 418-426. 